\title{
Classification and Categorization: Drawing the Line
}

\author{
Elin K. Jacob \\ School of Information and Library Science \\ University of North Carolina at Chapel Hill
}

\begin{abstract}
It is the intent of this paper to demonstrate that the processes of classification and categorization are actually two separate and distinct processes. The classical theory of categories is described, the major arguments against it are reviewed, and alternative approaches to the structure of categories are discussed. The apparent failure of the classical theory to account for the instability observed in category membership is attributed to the underlying assumption that the terms "classification" and "categorization" refer to the same process. The possibility that an interactive functional relationship exists between classification and categorization is advanced on the basis of the individual's need to communicate.
\end{abstract}

\section{INTRODUCTION}

Classification systems serve to organize knowledge. Through the ordered arrangement of classes of similar entities -- classes whose individual memberships are defined by a commonality both of characteristics and of name -- the process of classification imposes upon knowledge an empirical structure that explicitly delineates the relationship between these classes (Richmond 1960/1972). Austin points out that the imposition of such a classificatory superstructure "presupposes that knowledge possesses known boundaries, that the territory within these boundaries can be divided into separate autonomous states or main classes, and that every subject owes allegiance to one main class" (Austin, 1974, p. 372). He underscores the two primary functions of classification: Definition (through the determination of classes of entities that share characteristic attributes in common) and arrangement (through a systematic ordering of classes that expresses conceptual relationships within the overall structure).

Sharp (1965/1972) argues that an emphasis on the principle of class arrangement distinguishes the process of bibliographic classification from the process of classification in logic. A hierarchicallystructured bibliographic classification scheme defines and orders recorded knowledge through imposition of a fixed and superficial classificatory structure consisting of mutually exclusive superordinate classes and a series of divisions or subclasses nested within each superordinate. Thus a traditional, hierarchically-structured bibliographic classification scheme provides a conceptual structure or framework that physically represents the intellectual relationships between classes through the practical arrangement of documents within the library. Access -- the orderly process of storage and retrieval -- is facilitated through this physical embodiment of the classification scheme: Each document or entity is assigned a slot within one superordinate class and a unique notational symbol is provided as a marker identifying its physical location within the library. This symbol also serves to identify the intellectual content of the document by its relative position within the overall arrangement of the classificatory structure.

While current classification systems provide an efficient method for storing and retrieving physical "containers," the imposition of a fixed and arbitrary knowledge structure has been seen as counterproductive: The rigidity inherent in traditional classification schemes makes little allowance for the advancement of knowledge within individual disciplines; and recurrence of the 
same subordinate classes within disparate superordinates encourages the dispersion of related materials throughout both the physical and the intellectual structures (Austin 1974). Because current classification systems frequently employ a unitary approach to the organization of recorded knowledge, they have also been depicted as counterintuitive: The hierarchical structure they impose upon recorded knowledge is seen as failing to reflect the way individual users structure experiential knowledge of the real world (Molholt 1988).

Molholt emphasizes the inability of traditional structures to respond to idiosyncratic representations of knowledge when she writes of the "differences" that characterize individuals" perceptions of the world: "what terminology they use in describing the problem, how they structure the problem and identify its parts, and the sophistication of the information required to satisfy their queries" (1988, p. 99). From this perspective, the primary problem confronting the information industry is the need to facilitate effective interaction between the user and the system -- interaction that fosters access to appropriate and relevant materials within the constraints imposed by the cognitive representation of the individual. Interaction at this level can only proceed from an understanding of the relationship that exists between an external, artificial ordering of knowledge and the internal, mental representations of the user -- of the interaction between the intellectual structure imposed upon recorded knowledge by the classification system and the cognitive framework individuals impose upon their experiences of the world. Any preliminary understanding of this relationship must begin with the process of classification itself and its functional role in cognition.

\section{THE CLASSICAL THEORY OF CATEGORIES}

Foucault points out that the very possibility of introducing order within an empirical domain assumes that knowledge can be defined and arranged -- that knowledge is "at the same time describable and orderable" (Foucault, 1970, p. 158). The metaphysical structure imposed on the ordering of knowledge by traditional classification systems reflects the dual roles of definition and arrangement in its adherence to the classical theory of categories.

The classical theory of categories finds its origin in classical Greek philosophy. Aristotle maintained that one can only know a thing when one knows both its cause -- or explanation -- and that it cannot be otherwise. He held that the essence or essential properties that define a class of objects are often primary in that they have no other explanation than themselves. These selfexplanatory facts are seen as definitions; and it follows that the Aristotelian notion of a definition is a statement of the essence of a thing. Thus the Aristotelian theory of categories entails the assumption that necessary and sufficient properties determine category membership. The essential nature of a cow is primary -- or self-explanatory -- and consists of the knowledge that a cow simply is a cow, and that it cannot be otherwise. This is demonstrated in the following paraphrase of Aristotelian reasoning based on Aristotle's Parts of Animals, III 2, 664a8-11; 14, 674b5-14:

Why do cows have horns? Because they are deficient in teeth (so that the matter which would have formed teeth goes to make horns). Why are they deficient in teeth? Because they have four stomachs (and so can digest their food unchewed). Why do they have four stomachs? Because they are ruminants. Why, then, are cows 
ruminants? Simply because they are cows -- there is no further feature, apart from their being cows, which explains why cows are ruminants; the cause of a cow's being a ruminant is just its being a cow. (Bames, 1982, p. 34)

The term cow represents semantically the determining essence -- the essential property of "cowness" -- embodied in an entity belonging to the category cow. A cow is a cow simply because it is a cow.

Thus, according to the classical theory of categories, categorization is the process of systematically dividing up the world into a formalized, hierarchical ordering of classes or groups of entities which share some fundamental set of characteristics. Each class has a set of defining features -- a core of necessary and sufficient, or essential, properties -- that determines membership within that category. Furthermore, these defining features can be specified and each entity must exhibit the complete set of features to be accorded membership. As Brown observes, the classical view mandates that "any given thing is either in or out of the set; there are positive instances, all of which manifest the common characteristic, and noninstances which lack it. Membership is an either-or absolute because sets have clear boundaries" (Brown, 1979, p. 189)

These defining features are known as the intension (or meaning) of the category, and the entities comprising the category are its extensions (or referents). Membership within the class becomes a means of identifying or representing semantically the defining characteristics or essential properties embodied within an entity and of establishing the position of a particular entity within a predetermined and arbitrary hierarchical ordering of reality. Thus the classical theory of categories rests on the assumption that intension and extension are synonymous: Being a member (extension) of a particular category entails possession of an essential and defining character (intension).

The classical theory of categories is a powerful theory constructed on three propositions (Smith and Medin, 1981):

I. The intension of a category is a summary representation of an entire category of entities.

II. The set of essential features that comprise the intension of a category are individually necessary and jointly sufficient to determine membership within the category.

III. If a category (A) is nested within the superordinate category (B), the features that define category (B) are contained within the set of features that define category (A).

These three propositions provide the theoretical foundation for the classical theory of categories. Proposition I states that the sum of essential features (the intension) of a category is the union of those distinguishing features (the summary representation) which together identify the entire category and not merely a single instantiation, prototype, or subset of entities. Because all of the entities within a category must share the same set of distinctive characteristics, no one instantiation can be a better representative or a more typical example of that category than any other. 
Proposition II states that to be a member of a category an entity must exhibit all of the distinguishing characteristics (essential features) that comprise the intension, and that possession of the union of these characteristics or features is sufficient for determining membership within a category. This proposition is central in that it expresses the fundamental notions that, for many critics, have come to characterize the classical theory: Categories have rigid and fixed bounds, and there exists a binary, either/or relationship between an entity and a category. According to the classical theory, an entity either is, or is not, a member of a category.

Proposition III simply states that any subset of a superordinate category must exhibit not only those essential features which determine, or define, membership in the subset, but also those features which determine membership in the superordinate category.

\section{THE STRUCTURE OF CATEGORIES}

Because the classical theory of categories holds that an entity is a full and equal member of a category if it exhibits the set of essential characteristics or defining features that are both necessary and sufficient for category membership, it provides a simple and elegant theory of the internal structure of cognitive representations and semantic meanings. Historically, the classical approach has dominated the fields of both psychology and philosophy. Indeed, until very recently, research into the internal structure of cognitive categories did not challenge the assumptions underlying the classical theory, but tended to concentrate on the process of concept acquisition, adopting the classical approach as the accepted model of category structure. Thus, in 1920, the psychologist Hull wrote of the child's discovery of meaning in the word dog:

The "dog" experiences appear at irregular intervals.... At length the time arrives when the child has a "meaning" for the word dog. Upon examination this meaning is found to be actually a characteristic more or less common to all dogs and not common to cats, dolls and "teddy-bears." (Hull, 1920, pp. 5-6; cited in Brown, 1979 , p. 188)

Hull represented the category dog as a proper set whose essential nature, while not formally marked, is nonetheless available to, and ultimately recognized by, the child. Brown (1979) points out that Hull failed to specify a particular characteristic or set of characteristics that would define the category dog for the child. "Had he done so, he might have discovered that 'natural categories' are not usually definable in the way he supposed" (Brown, 1979, p. 189).

Although Hull's research was centered on the process of concept formation, his theoretical approach obviously assumed the classical criteria for category membership. In the 1950 s, the work of Bruner, Goodnow, and Austin (1956) retraced much the same ground covered by Hull in 1920, albeit in greater depth. Their interest, like that of Hull, centered on the development of concepts and on the identification of those cognitive strategies employed by subjects in their attempts to discover the defining attribute of a proper set -- the set of necessary and sufficient criteria as predetermined by the experimenter (Brown, 1979). Here, too, assumptions about the nature of category structure were firmly grounded in the classical model. 
More recently, arguments against the classical theory have focused attention on the assumptions expressed in Propositions I and II. Based in large part on research exploring typicality effects and the nature of family resemblances (McCloskey and Glucksberg, 1978; Rips, Shoben, and Smith, 1973; Rosch, 1973, 1975; Rosch and Mervis, 1975), the charge has been leveled that membership within a category is not determined by a set of essential features whose union is both necessary and sufficient, but that categories often have member entities who do not share a specifiable set of essential characteristics. Research has demonstrated that the membership of a category generally exhibits graded typicality effects, or graded structure: Individuals frequently judge particular member entities as more representative, or prototypical, of a category than other, less typical members (McCloskey and Glucksberg, 1978; Rips, Shoben, and Smith, 1973). Other findings have indicated that the cognitive boundaries marking a category are not fixed and determinate: In point of fact, there may be no clear boundaries separating members of a category from nonmembers (Rosch and Mervis, 1975).

The view that categories frequently exhibit "fuzzy" boundaries because they are organized by the principle of "family resemblances" evolved from the philosophy of Wittgenstein, whose description of the category games typifies the notion of family resemblances. Wittgenstein drew an analogy between those similarities of build, features, color of eyes, etc., that characterize a family and the "complicated network of similarities overlapping and criss-crossing: sometimes overall similarities, sometimes similarities of detail" (1953, p. 328) that characterize the category game: "Consider for example the proceedings that we call 'games'. I mean board-games, card-games, ball-games, Olympic games, and so on. What is common to them all?... For if you look at them you will not see something that is common to all, but similarities, relationships, and a whole series of them at that" (Wittgenstein, 1953, pp. 328-329).

Research findings reported by Rosch and Mervis (1975) supported their contentions that "formal criteria are neither a logical nor psychological necessity" (p. 603) and that the notion of family resemblance could provide a viable alternative to the concept of "common criterial attributes" (p. 603). But Smith and Medin (1981) dismissed Wittgenstein's analysis on the basis that it "is nothing like a principled disproof of the classical view; it is instead an empirical argument about the observed rate of progress of a theoretical approach to concepts" (p. 31)

Wittgenstein's argument and the research it precipitated raised significant questions about the existence of a central core of essential properties that are both necessary and sufficient for category membership. The observation that many categories exhibited graded typicality efferts, or "family resemblances", was interpreted as particularly troublesome for adherents to the classical theory. Armstrong, Gleitman, and Gleitman (1983) attempted to discredit the arguments advanced by proponents of family resemblance theory by demonstrating that the graded typicality effects interpreted as empirical support for the notion of family resemblance were actually produced in subject responses to all categories. The authors concluded that graded typicality responses to representatives of well-defined, either/or categories such as odd number, even number, and female, as well as purportedly "fuzzy" categories such as vegetable and fruit, raised serious problems for the notion of family resemblance: 
The prior literature has shown that exemplars from various categories receive graded responses, in a variety of paradigms. But graded responses to exemplars of such categories as fruit do not constitute evidence for the family resemblance structure of these categories without--at minimum--a further finding: all-or-none responses to exemplars of categories that are known to have definite, all-or-none, descriptions and whose all-or-none descriptions are known to be known to the subjects. And this is precisely what we failed to find. (Armstrong, Gleitman, and Gleitman, 1983, p. 291).

While their work is frequently cited as empirical evidence supporting the classical theory of categories (Markman, 1989; Medin, 1989; Medin and Smith, 1984; Rey, 1983), Armstrong, Gleitman, and Gleitman claim that the main purport of their research findings points, instead, to the conclusion that there are methodological problems that jeopardize current studies of category structure: "[O]ur results indicate that certain techniques widely used to elicit and therefore elucidate the structure of such categories are flawed. This being so, the study of conceptual structure has been put on an experimental footing, and the structure of those concepts studied by current techniques remains unknown" (Armstrong, Gleitman, and Gleitman, 1983, p. 291).

But the research conducted by Armstrong, Gleitman, and Gleitman fails to address one of the more damaging criticisms leveled at the classical theory of categories: That membership within a category is not determined by a set of essential features whose union is both necessary and sufficient for category membership. The basis for this contention is the inability of subjects to provide a list of defining or essential features that are both necessary and sufficient for all members of a class (Hampton, 1979; Rosch and Mervis, 1975). This accusation, supported as it is by the evidence of graded typicality effects (McCloskey and Glucksberg, 1978; Rips, Shoben, and Smith, 1973; Rosch, 1973) and family resemblance structures (Rosch and Mervis, 1975), strikes at the theoretical heart of the classical view and engenders two very serious criticisms: The findings that certain member entities are judged to be more representative or typical of a category than other, less typical members (McCloskey and Glucksberg, 1978) directly contradicts the classical assumption that, because the members of a category share the same set of essential features that define category membership, no one member can be more typical or a better representative of the category than any other member (Proposition I); and the notion that there may be no well-defined and determinate boundary separating members of a category from nonmembers (Rosch and Mervis, 1975) controverts the assumption that, because categories exhibit rigid and fixed boundaries, there is an explicit, either/or relationship between an entity and a category (Proposition II).

\section{RECENT APPROACHES TO THE STUDY OF CATEGORIES}

Within the past twenty years, there has been a prodigious amount of research focusing on the process of categorization and the cognitive structure of categories. Although the work of Eler.nor Rosch (1973) is frequently cited as the catalytic force precipitating this onslaught of interest in the nature of categorization, Rosch (1988) identifies an article by psychologist Roger Brown (1958) as the seminal piece directly influencing her own work and, indirectly, the work of those who have followed her lead. Brown's essay pointed out that, while we may think of an object as having a 
single identifying marker or semantic label indicating category membership, there are, in fact, a variety of different names by which an entity is generally known:

The dog out on the lawn is not only a dog but is also a boxer, a quadruped, an animate being; it is the landlord's dog, named Prince. How will it be identified for a child? Sometimes it will be called a dog, sometimes Prince, less often a boxer, and almost never a quadruped, or animate being. Listening to many adults name things for many children, I find that their choices are quite uniform... How are these choices determined and what are their consequences for the cognitive development of the child? (Brown, 1958, p. 14)

Based on available research, Brown concluded that, in the process of identifying objects in the physical world, children are initially presented with the particular label that embodies the greatest utility -- the common name: "It seems likely that things are first named so as to categorize them in a maximally useful way.... The same referent may have its most useful categorization on one level (Prince) for one group (the family) and on another level (dog) for another group (strangers). The categorization that is most useful for very young children (money) may change as they grow older (dime and nickel)" (Brown, 1958, p. 20). Obviously, a dog might variously be categorized as a mammal, an animal, a pet, a possession, a friend, or an enemy. The important distinction illustrated here is that the concept dog can be identified within any of a number of different categories that will reflect the variety of ways in which individuals cognitively represent the category dog.

Following Brown's line of reasoning, Rosch, Mervis, Gray, Johnson, and Boyes-Braem (1976) argued against the traditional approach to categorization as an arbitrary process:

One of the most basic functions of all organisms is the cutting up of the environment into classifications by which nonidentical stimuli can be treated as equivalent.... [I]t has been the tendency both in psychology and anthropology to treat segmentation of the world as originally arbitrary and to focus on such matters as how categories, once given, are learned or the effects of having a label for some segment. (Rosch et al., 1976, 382-383)

Rosch et al. proposed that individuals perceive structure in the world because attributes of objects do not occur independently but in associations: "Some pairs, triples, or ntuples are quite probable, appearing in combination sometimes with one, sometimes with another attribute; others are rare; others logically cannot or empirically do not occur" (1976, p. 383). Categories of equivalent entities in the concrete, physical world are related to each other in a hierarchical taxonomic system based on class inclusion. But within such a taxonomy there is a basic level of inclusiveness at which categories, like those represented by Brown's (1958) notion of common names, are of greatest utility. This basic level category is "most cognitively efficient" (Mervis and Rosch, 1981, p. 92) in that it carries the greatest amount of information about category members. Furthermore, Rosch et al. contended, it is at this level that a category carries the greatest cue validity: That is, categories at the basic level distinguish the maximum number of possible attributes that are common across members while embodying the least number of attributes that may be shared across categories. They concluded: 
The correlational structure of the environment, modified by selective ignorance and exaggeration of the attributes and structure of that environment, are mirrored in categorization systems. Segmentation of experience occurs to form basic levels which maximize the differentiability of categories. For categories of concrete objects, basic objects are the most general classes at which attributes are predictable, objects of the class are used in the same way, objects can be readily identified by shape, and at which classes can be imagined. Basic objects should generally be at the most useful level of classification. (Rosch et al., 1976, p. 435)

As Markman (1989) and Smith and Medin (1981) have pointed out, the notion of basic level categories whose members are maximally distinguishable from members of other categories has generated its share of criticism -- criticism that focuses heavily on the need to identify that level of abstraction at which a category is to be considered most basic. But the argument for a basic level within category structure was based, in large part, on the assumption that basic level categories are those which "best mirror the correlational structure of the environment" (Rosch et al., 1976, p. 385). Correlation with the environment was similarly advanced as an argument for the earlier contention that family resemblance offered a viable alternative to a set of necessary and sufficient criterial features in determining category membership: "In natural categories of concrete objects,... family resemblance should coincide [with typicality effects] rather than conflict since it is reasonable that categories tend to become organized in such a way that they reflect the correlational structure of the environment in a manner which renders them maximally discriminable from each other" (Rosch and Mervis, 1975, p. 575).

The notions of basic level categories and family resemblance rely heavily on the assumption that the composition of categories reflects the underlying structure inherent in the physical world, a structure perceived by the individual as recurring patterns or associations of attributes. Correlation with the structure of the environment, both within and between categories, was proposed to account not only for the composition of categories, but for the phenomenon of graded structure. From this perspective, graded structure within a category reflects the degree to which member entities represent the pattern of correlations that determines category membership (Rosch, 1978; Rosch and Mervis, 1975).

But Barsalou contends that, while individuals' representations "may reflect environmental structure to some extent, it appears that their ability to manipulate knowledge allows them to construct representations that go far beyond those that reflect this structure" (Barsalou, 1987, $p$. 116). Furthermore, Barsalou argues, environment cannot account for variations in graded structure produced by context, explain the role played by ideals in the determination of graded structure or account for the presence of graded structure in goal-derived categories, which do not exhibit correlational structure with the environment.

Barsalou (1987) maintains that the role of graded structure, or typicality judgments, is central to the formulation of a theory of categorization. He points out that observations of graded structure do not describe cognitive structure but the behavior of individuals in assigning category membership based on typicality. Thus typicality judgments are applicable not only to category members, but to nonmembers as well: Subjects can, and do, rank entities as to how well they represent "nonmembership" in a category (Barsalou, 1983). The notion of graded structure refers 
to a "continuum of category representativeness, beginning with the most typical members of a category and continuing through its atypical members to those nonmembers least similar to category members" (Barsalou, 1987, p. 102).

Graded structure exhibits a high degree of instability, varying both between and within individuals as a function of time and context. Contradicting reported findings of values in excess of. 9 for agreement on graded structure between subjects (Rosch, 1975; Armstrong, Gleitman, \& Gleitman 1983), Barsalou claims that "values of subject agreement for typicality generally average around.45... [W]e have always obtained average values across categories of around.50, with these averages generally ranging from around.30 to.60" (1987, p. 108). Furthermore, typicality judgments within individuals exhibit instability across time: "Within-subject reliability decreased from around.92 at a delay of one hour, to around.87 at a delay of one day, and to around .80 at delays of one, two, and four weeks" (Barsalou, 1987, p. 111-112). More typical members, as well as the more atypical, appear to be most stable, while those members which ranked as only moderately typical exhibit the greatest amount of instability. Consonant with these findings are the results of research conducted by McCloskey and Glucksberg (1978), who found that typicality ratings used in the identification of category membership were inconsistent for items of intermediate typicality, varying not only between subjects but within individuals across time.

The pervasiveness of graded typicality effects in the membership rankings of categories, in association with the findings of substantial instability in the graded structure of categories, indicates that the information used by individuals to determine category membership is itself highly variable. Working from the assumption that graded structure is produced in working memory by a process of similarity comparison between exemplars and the concepts representing a category at a particular time and in a particular context, Barsalou proposes that "graded structure changes across contexts because different category concepts are used in different contexts for the same category" (1987, p. 117). Barsalou carefully points out that use of the term concept in this context does not refer to "mental categories" (Armstrong, Gleitman, \& Gleitman, 1983, p. 264) or to the definitional criteria that determine category membership in the classical theory of categorization. Rather, the construct concept refers to that specific information which serves to represent the intension of a category at a particular point in time. Furthermore, Barsalou assumes "that the concept used to represent a category on a particular occasion contains (1) information that provides relevant expectations about the category in that context, and (2) information that provides relevant expectations when interacting with the category in most contexts. These two kinds of information correspond to context-dependent and context-independent information" (Barsalou, 1987, p. 116).

Thus graded structure can vary both between and within individuals because different concepts are used to represent the same category at different times and in different contexts: Instability in category membership and typicality judgments both within and across subjects would represent variations in the concepts used to represent a category as a function of context, goals, and/or recent experience. Furthermore, variation in the concepts used to represent a category across contexts would reflect not only changes in a concept's properties, but alterations in the associations drawn between concepts. 
The classical theory of categories assumes that there are invariant concepts -- necessary and sufficient criteria -- that individuals use to determine category membership. The observation of graded structure in both well-defined, either/or categories, such as odd number and even number, and prototype or "family resemblance" categories, such as fruit and vegetable, cannot be summarily dismissed as failing of methodology because it does not fit the classical model. Given the apparent variability of concepts used to represent categories both within and across individuals, the search for structure in the composition of categories may be little more than an exercise in futility. Barsalou (1987) contends that it is the process of category construction, not the structure of categories, that should absorb research efforts:

... the concepts that theorists "discover" for categories may never be identical to an actual concept that someone uses. Instead, they may be analytic fictions that are central tendencies or idealizations of actual concepts. Although such theoretical abstractions may be useful or sufficient for certain scientific purposes, it may be more fruitful and accurate to describe the variety of concepts that can be constructed for a category and to understand the process that generates them. (p. 120)

Medin and Smith (1984) have claimed that the results reported by Armstrong, Gleitman, and Gleitman (1983) provide support for the notion that "a prototype identification procedure can coexist with a classical core" (Medin and Smith, 1984, p. 120). They propose that a concept of essential or core features can be used as a means for justifying categorizations based on a typicality or prototype identification procedure, as suggested in their earlier work (Smith and Medin, 1981). In an attempt to save the classical theory and the assumption of a core concept of defining features, with little or no support from the arena of empirical research and relying admittedly on their own intuitions, Medin and Smith suggest that individuals "approach the world as if it conformed to the classical view" and that, on this basis, the classical view may serve "as the layperson's metatheory of concepts" (1984, p. 123). In the face of research indicating that individuals cannot define those essential features that would comprise such a core concept (Hampton, 1979; Rosch and Mervis, 1975), Medin and Smith (1984) advance Putnam's (1973, 1975) philosophical theory of the division of linguistic labor as support for their notion of a "metatheory of concepts": individuals might reconcile their inability to define the essential properties of a category by assuming that these features are present, but hidden from view, and that they can be ascertained by domain experts who have the requisite knowledge to perceive them.

Obviously, theoretical approaches to the study of categories are in a state of flux. Apart from attempts to discredit the reliability of unfavorable research findings (Armstrong, Gleitman, \& Gleitman, 1983) or to salvage some semblance of the classical theory of categories through compromise or amalgamation (Medin, 1989; Medin and Smith, 1984; Smith and Medin, 1981) or the philosopher Putnam's $(1973,1975)$ more elaborate and eminently more successful efforts to reintroduce the classical theory of categories as the foundation for a linguistic theory of meaning, psychologists appear to have reluctantly abandoned the classical approach without reaching consensus on a suitable candidate to take its place.

While many philosophers and psychologists are reluctant to relinquish the classical theory of categories precisely because it is a very powerful and elegant theory, findings from empirical research demand the development of a theoretical framework that can accommodate graded 
typicality effects, as the classical theory cannot. Various approaches have been advanced that might reasonably account for the existence of graded structure within even the most well-defined of either/or categories. But the obvious utility of the classical theory is not easily relinquished. Rosch's reliance on the notion of the correlational structure of the environment (Rosch and Mervis, 1975; Rosch et al., 1976) in the arguments both for family resemblance and basic level categories is an obvious case in point. She contends that cognitive categories mirror the structure of the environment and reflect the probabilistic nature of co-occurring atrributes perceived in the physical world (Rosch, 1978). This argument, while it may provide a reasonable basis for instability of categories across subjects, cannot adequately account for short-term instability of categories within subjects or for the graded structure observed in abstract or goal-derived categories (Barsalou, 1987). Furthermore, the notion of a universal correlational structure that mirrors the environment begins to sound like an echo of the classical theory's insistence of a set of essential features in that it mandates an external and universal standard against which to measure the internal structure of cognitive categories.

\section{THE DISTINCTION BETWEEN CLASSIFICATION AND CATEGORIZATION}

The very fact that researchers and theorists have not been able to reach consensus on the nature of cognitive categories may devolve, to some extent, from a general confusion in the terminology used to describe the process -- or processes -- under investigation. Throughout the literature on categorization, the terms classification and categorization are often employed as synonyms referring to what is purported to be one and the same process. Rosch et al. provide an illustrative example of the confusion that exists between these two terms:

... one purpose of categorization is to reduce the infinite differences among stimuli to behaviorally and cognitively usable proportions. It is to the organism's advantage not to differentiate one stimulus from others when that differentiation is irrelevant for the purposes at hand. The basic level of classification, the primary level at which cuts are made in the environment, appears to result from the combination of these two principles; the basic categorization is the most general and inclusive level at which categories can delineate real-world correlational structures. (1976, p. 384. Emphasis added.)

Gardner (1985) is more blatant about the relation of synonymy that is assumed between classify and categorize, between classification and categorization, or, for that matter, between classes and categories: "By the middle of this century, a certain position had become entrenched as the "right way' to think about categories, concepts, and classification (a trio of terms I siall use here interchangeably)" (p. 340). This confusion between the various linguistic forms of classify and categorize -- between class and category -- is further compounded by introduction of the term concepts to represent cognitive categories, the characteristics that identify these categories, and the complex ideas that are formed from the conjunction of categories. Unfortunately, many dictionaries identify classify as a synonym for the verb categorize. ${ }^{1}$ But even a cursory review of the philosophical and psychological literature on categorization strongly suggests that this

1. The fact that the relationship on synonymy is unidirectional -- that categorize is not offered as a synonym for classify -- is intriguing. A detailed study of the etymological derivations of these two verbs might contribute another dimension to the present argument. 
terminological imprecision has served to obscure the realization that researchers are, in fact, dealing with two closely related but nonetheless separate and distinct processes.

The process of classification involves the systematic assignment of entities to groups or classes according to an established set of principles. Classification entails a one-for-one slotting of objects, events, or properties, based upon the apperception of a core of necessary and sufficient characteristics, into mutually exclusive classes within the hierarchical structure imposed by an arbitrary and predetermined ordering of reality. Categorization, on the other hand, refers to the process of dividing the world of experience into groups -- or categories -- whose members bear some perceived relation of similarity to each other. In contrast to the process of classification, the process of categorization entails neither that membership within a category is determined by the apprehension of a set of definitive characteristics nor that inclusion within one category prohibits membership within any other category. It is this recognition of similarities between otherwise unlike entities and the subsequent identification of categories that permits the individual to discover order within an otherwise complex and chaotic environment.

The process of classification is best exemplified by taxonomy, the science of classification. The objective of scientific, or analytical, taxonomy is the orderly and systematic organization of knowledge about the biological world. This is achieved through the institution of a hierarchical ordering of mutually exclusive superordinate and subordinate classes in accordance with a set of established and universally accepted principles and laws.

Based on this system of laws, each entity is evaluated, its essential or defining features are determined, and it is assigned to a unique group or class within the hierarchy. Each class is assigned a unique Latin name, or label, that specifies the set of necessary and defining characteristics that distinguish this class from all other classes in the hierarchy. Each class has clear and well-defined boundaries: A given entity must display the full complement of essential features to be included in a particular class and only those entities that display this set of defining characteristics are identified by the unique label assigned to that class. In turn, this label, which is universally employed to identify the members of a particular class, serves as a marker that provides access to information about the entities within that class. In the plant kingdom, for instance, a plant that is widely distributed throughout various regions of the world will be assigned a scientific name by which it is recognized wherever it occurs, and, because all members of the same class must display the essential features that identify that class, each plant so named will be recognizable as belonging to the same species. In this manner, scientific classification establishes the stability of the nomenclature through the aegis of a universally accepted language that facilitates the transmission of knowledge across the barriers of natural language.

The process of taxonomic classification epitomizes the classical theory of categories: A biological entity is a full and equal member of a particular class if it exhibits those essential characteristics or defining features that are both necessary and sufficient for category membership. Scientific classification, like the classical theory of categories, rests on the assumption that intension and extension are synonymous, that being a member (extension) of a particular class entails possession of the essential and defining character (intension) that marks that class as unique. 
While the process of classification is both rigorous and absolute in that it mandates that an entity either is or is not a member of a particular class, the process of categorization is flexible and creative in that it facilitates associations between and among entities based upon the recognition of similarities. Medin (1989) observes that, in the treatment of patients, clinical psychologists cannot approach each individual as a unique entity precisely because the determination of "absolute uniqueness imposes the prohibitive cost of ignorance" (p. 1469). By grouping patients according to observable similarities, clinicians and diagnosticians can access knowledge based on previous experiences to predict the utility of alternative approaches to therapy and can apply that knowledge in the treatment of the individual. In this manner, the apprehension of similarity brings knowledge: Repeated experience with patients who present common symptoms permits clinicians to cumulate and synthesize information about a diagnostic category and to expand the scope of their knowledge accordingly. Medin concludes that the process of categorization, while disdaining the unique, encourages diversity: "Ironically, the only case in which categorization would not be useful is where all individuals are treated alike" (p. 1469).

Categorization performs a fundamental function in the process of cognition. By recognizing similarities between potentially dissimilar entities, the individual is enabled to form theories, or models, of her environment that allow her to extend to new encounters the generalizations garnered from past experience. Markman describes categorization as a "fundamental cognitive process" and points out that, without the ability to recognize the similarities that exist between otherwise dissimilar entities, "we would be overwhelmed by the complexity of our environment.

Categorization, then, is the means of simplifying the environment, of reducing the load on memory, and of helping us to store and retrieve information efficiently" $(1989$, p. 11).

Like the classes nested within a hierarchical classificatory system, cognitive categories do not exist in isolation but are frequently combined into hierarchical relationships that include specific instances within the structure of broader or more general superordinate categories. But, unlike the classes in a classificatory system, categories do not exhibit fixed boundaries demarcated by a set of essential characteristics. It would be a Herculean task to define a set of necessary and sufficient features that would identify the category tree uniquely. The idea of a tree is easily understood by most people. It is a woody plant, often deciduous but sometimes evergreen, of a considerable height and frequently, but by no means always, with a single stem or trunk. But what is it that determines "of a considerable height"? There are "trees" such as lovely Chionanthus virginicus, or "fringe tree", that are typically multi-stemmed and only rarely exceed twelve to fifteen feet in height, a height that, by many standards, would not be likely to be judged "considerable." In contrast, Acer palmatum dissectum, the "cutleaf Japanese maple," is generally single-stemmed, but may not be over six to eight feet in height at maturity. Furthermore, there are plants that are categorized as shrubs, such as Chamaecyparis obtusa nana gracilis, that are single-stemmed, while other shrubs, such as Magnolia stellata (the "star magnolia") or Corylopsis glabrescens ("fragrant winterhazel"), may reach eighteen to twenty feet, or more, in height. Obviously, the term tree signifies a category of entities whose boundaries are loosely defined and highly variable. To say that a certain plant is a tree is to assign it to a category whose boundaries, though generally defined, are frequently determined by the speaker: the woody plants called "trees" by the Ozark mountain woman might be categorized as no more than "scrub" by a visitor from North Carolina. 


\section{CONCLUSION}

In his critique of Smith and Medin's Categories and Concepts (1981), philosopher Georges Rey (1983) identifies four primary functions that categories perform: The stability function encourages consistency of mental representations both within and across individuals; the linguistic function provides meaning for linguistic terms through relations of synonymy, antinomy, semantic implication, and translation; the metaphysical function defines or determines the validity of a taxonomic identification, where such an identification is held to be metaphysically true if it is true in all possible worlds; and finally, the epistemological function assures that the identification of an entity within a category is epistemologically possible -- that it is compatible with the individual's extended knowledge of the world.

Acknowledging their debt to Rey's philosophical insights, psychologists Medin and Smith (1984) offer their own set of four functions, or roles, performed by concepts, where the term concept is intended to refer to the mental representation of a simple category. Simple categorization is described as the process that determines whether or not an entity is an instance of a particular category such as dog. Complex categorization is the function that determines membership within a conceptually complex category, such as mean dog. Linguistic meaning, the only function attributed to concepts by Medin and Smith that bears any resemblance to the category functions described by Rey, explains semantic relationships between words, as in the relation of synonymy between dog and canine or that of antimony between dog and cat. Components of cognitive states, the final role described by Medin and Smith, represents what they term "the critical component" of belief: As the components of cognitive states, "concepts are what provide a cognitive explanation of complex thought and behavior" (Medin and Smith, 1984, p. 114). Thus the concepts or simple categories mean, dog, and bite interact to produce the conceptual belief that mean dogs bite.

Although Medin and Smith (1984) credit Rey (1983) as the primary source for the cognitive functions they attribute to categories, the divergence between these two approaches is significant. The obvious reliance of Medin and Smith on an epistemological interpretation of categories (apprehensions of reality by the individual) to the exclusion of metaphysical concerns (the underlying nature of reality that extends across all possible worlds) underscores the conflict that pervades recent research into the process of categorization and the nature of categories. The classical theory of categories assumes that extension equals intension -- that being a member of a particular category entails possession of an essential and defining character that extends across all possible worlds. But research has demonstrated that the classical theory does not have sufficient explanatory power to account for the instability in category membership that reflects the variety and flexibility with which individuals create cognitive representations of reality.

While various theories have been advanced to account for the instability of categories, it seems obvious that knowledge of the cognitive roles performed by categories cannot proceed until researchers effectively separate the process of classification from that of categorization. The confusion that suffuses research into the cognitive nature of categories is due, in large part, to a pervasive failure to acknowledge that classification and categorization are distinct processes -- that "classes" function within a rigidly fixed and universal order exemplified by the analytical process of classification, while "categories" function at the idiosyncratic, cognitive level of the individual through the context-based process of categorization. 
Their findings of graded structure in the representation of such well-defined, either/or categories as odd number and even number led Armstrong, Gleitman, and Gleitman (1983) to conclude that the notion of graded structure was at once unreliable and meaningless and that the experimental techniques generally employed by psychologists to investigate the nature of categories must be flawed. Barsalou argues that this interpretation rests on the classical assumption that

there are invariant cognitive structures associated with categories that we should be trying to discover. According to this view, people's representations of categories exist in some clear-cut way, and our job as cognitive scientists is, first, to develop empirical means for identifying these structures and, second, to develop theoretical means for discussing them.... Invariant representations of categories do not exist in human cognitive systems. Instead, invariant representations of categories are analytic fictions created by those who study them." (Barsalou, 1987, p. 114)

The analytical organization of scientific taxonomy and the artificial conceptual structure imposed on the ordering of knowledge by hierarchically-structured bibliographic classification schemes have much in common with the classical theory of categories. Both are rigidly constrained by a logical but nonetheless arbitrary ordering of the world. The individual, however, is under no such constraint in the cognitive ordering of reality. Rather, as Keil (1987) points out, individuals may be constrained by a communicative principle. The demands placed on an individual by the need to communicate an idiosyncratic representation of the world can force the adoption of a category structure that is more analytical and therefore more stable, or consistent, across individuals:

The need to share conceptual knowledge in communication also places constraints on concepts, constraints that will often push the conceptual structure toward a more analytic form that defies typicality distributions. People attempt to reach a common ground or consensus by tacitly agreeing on certain common values or dimensions for organizing the concepts involved. (Keil, 1987, p. 193)

The principle of communicative constraint is important because of the implications it holds for understanding the relationship between classification and categorization. Given the instability of category structure both within and between individuals, the need to communicate may mandate that the categories produced by the individual at the idiosyncratic, cognitive level be represented in terms of categories that function at a more universal level -- a level that would approximate the classes constructed according to the classical theory of categories. Understanding how the individual uses categories to structure his own knowledge is fundamental. But equally as important is an understanding of how these categories may be structured, or restructured, by the need to communicate that knowledge to others.

The possibility that categories function in accordance with the classical theory at one level while exemplifying aspects of instability, graded structure, and fuzzy boundaries at another should encourage researchers to set aside, for the moment, the emphasis on category structure and to focus their attention, instead, on the generation of cognitive categories and on the role(s) performed by these idiosyncratic categories in the processes of cognition and communication. Keil's recognition that the need to communicate information can force individuals to restructure their mental representations in accordance with a set of essential features or "common values or dimensions" 
(Keil, 1987, p. 193) points the way toward an enhanced understanding of how the idiosyncratic, personalized categories produced by the individual interact with the universal, analytical categories used in classification.

The important question here is not "What is the structure of categories?", but "Does the individual, given the constraints imposed by the need to communicate, restructure internalized representations of reality -- those idiosyncratic cognitive categories -- to reflect a fixed and arbitrary external ordering of knowledge? And, if so, how?" The answers to this complex question should enhance understanding of the functional relationship that exists between classification, cognition, and category formation. It is quite possible that they will also contribute to an awareness of the role that individual cognitive categories play in judgments of relevance or, just as importantly, provide direction for improving access to materials organized in accordance with current classification schemes.

\section{WORKS CITED}

Armstrong, S. L., Gleitman, L. R., \& Gleitman, H. (1983). What some concepts might not be. Cognition, 13, 263-308.

Austin, D. (1974). A conceptual approach to the organization of machine-held files for subject retrieval. In J. A. Wojciechowski (Ed.), Conceptual basis of the classification of knowledge (pp. 371-398). Pullach/Munchen: Verlag Dokumentation.

Barnes, J. (1982) Aristotle. Oxford: Oxford University Press.

Barsalou, L. W. (1983) Ad hoc categories. Memory and Cognition, 11, 211-227.

Barsalou, L. W. (1987). The instability of graded structure: Implications for the nature of concepts. In U. Neisser (Ed.). Concepts and conceptual development: The ecological and intellectual factors in categorization (pp. 101-140). Cambridge, England: Cambridge University Press.

Brown, R. (1958). How shall a thing be called? Psychological Review, 65, 14-21.

Brown, R. (1979). Cognitive categories. In R. A. Kasschau and C. N. Cofer (Eds.), Psychology's second century: enduring issues (pp. 188-217). New York: Praeger.

Bruner, J.S., Goodnow, J.J., \& Austin, G.A. (1956). A study of thinking. New York: Wiley.

Foucault, M. (1970). The order of things. New York: Random House

Gardner, H. (1987) The mind's new science: A history of the cognitive revolution. New York: Basic Books, Inc.

Hampton, J. A. (1979) Polymorphous concepts in semantic memory. Journal of Verbal Learning and Verbal Behavior, 18, 441-461.

Hull, C. L. Quantitative aspects of the evolution of concepts. Psychological Monographs, 28, Whole No. 123. [Cited in Brown, 1979]

Keil, F. C. (1987). Conceptual development and category structure. In U. Neisser (Ed.). Concepts and conceptual development: The ecological and intellectual factors in categorization (pp. 175-200). Cambridge, England: Cambridge University Press.

McCloskey, M.E., and Glucksberg, S. (1978). Natural Categories: Well defined or fuzzy sets?

Memory and Cognition, 6, 462-472.

Markman, E.M. (1989). Categorization and naming in children: Problems of induction. Cambridge: MIT Press.

Medin, D.L. (1989). Concepts and conceptual structure. American Psychologist, 44, 1469-1481. 
Medin, D.L., and Smith, E.E. (1984). Concepts and concept formation." Annual Review of Psychology, 35, 113-138.

Mervis, C.B., and Rosch, E. (1981). Categorization of natural objects. Annual Review of Psychology, 32, 89-115.

Molholt, P. (1988). Research issues in information access. In A.J. Matthews (Ed.), Rethinking the library in the information age: Vol. II (pp. 93-113). Washington, D.C.: U.S. Department of Education

Putnam, H. (1973). Meaning and reference. Journal of Philosophy, 70, 710-711.

Putnam, H. (1975). The meaning of 'meaning'. Mind, language and reality: Philosophical papers, Vol. 2. Cambridge: Cambridge University Press.

Rey, G. (1983). Concepts and stereotypes. Coginition, 15, 237-262.

Richmond, P.A. (1972). Some aspects of basic research in classification. In A.F. Painter (Ed.), Reader in classification and descriptive cataloging (pp. 129-153). Washington, D.C.: NCR/ Microcard Editions. (Reprinted from Library Resources and Technical Services, 1960, 4, pp. 139-147)

Rips, L.J., Shoben, E.J., \& Smith, E.E. (1973). Semantic distance and the verification of semantic relations. Journal of Verbal Leaming and Verbal Behavior, 12, 1-20.

Rosch, E. (1973) Natural categories. Cognitive Psychology, 4, 328-350.

Rosch, E. (1975). Cognitive representations of semantic categories. Journal of Experimental Psychology: General, 104, 192-233.

Rosch, E. (1978) Principles of categorization. In Rosch, E. and Lloyd, B.B. (Eds.), Cognition and Categorization. Hillsdale, NJ, Lawrence Erlbaum Associates, 27-48.

Rosch, E. (1988). Coherences and categorization: A historical view. In F.S. Kessel (Ed.), The development of language and language researchers: Essays in honor of Roger Brown. Hillsdale, NJ: Lawrence Erlbaum Associates.

Rosch, E. and Mervis, C.B. (1975). Family resemblances: Studies in the internal structure of categories. Cognitive Psychology, 7, 573-605.

Rosch, E., Mervis, C.B., Gray, W., Johnson, D. \& Boyes-Braem, P. (1976). Basic objects in natural categories. Cognitive Psychology, 7, 382-439.

Sharp, J. (1972). Classification: Current functions and applications to the subject analysis of library materials. In A. F. Painter (Ed.), Reader in classification and descriptive cataloging (pp. 57 67). Washington, D.C.: NCR/Microcard Editions. (Reprinted from John Sharp, (1965), Some fundamentals of information retrieval (pp. 20-47). London: Andre Deutsch)

Smith, E. and Medin, D. (1981). Categories and concepts. Cambridge: Harvard University Press. Wittgenstein, L. (1953). Philosophical investigations. New York: Macmillan. 
Jacob, E. (1991). Classification and Categorization: Drawing the Line. 2nd ASIS SIG/CR Classification Research Workshop, 63-80. doi:10.7152/acro.v2i1.12548 